\title{
Software Engineering and Environment
}

An Object-Oriented Perspective 


\title{
Software Science and Engineering
}

\author{
Series Editor: Richard A. DeMillo
}

Purdue University, West Lafayette, Indiana

High-Integrity Software

Edited by C. T. Sennett

Software Engineering and Environment: An

Object-Oriented Perspective

Phillip C.-Y. Sheu

Software Reuse: Guidelines and Methods

James W. Hooper and Rowena O. Chester

Studies in Computer Science: In Honor of Samuel D. Conte

Edited by John Rice and Richard A. DeMillo

A Continuation Order Plan is available for this series. A continuation order will bring delivery of each new volume immediately upon publication. Volumes are billed only upon actual shipment.

For further information please contact the publisher. 


\title{
Software Engineering and Environment
}

\section{An Object-Oriented Perspective}

\author{
Phillip C.-Y. Sheu
}

University of California, Irvine

Irvine, California

Springer Science+Business Media, LLC 


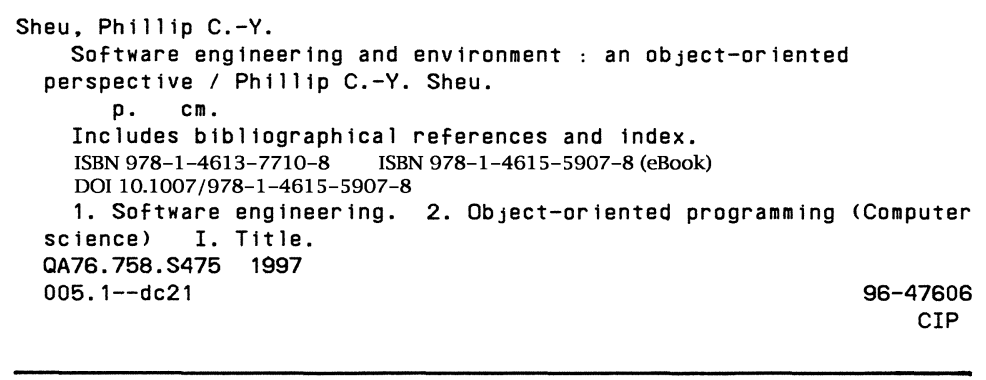

ISBN 978-1-4613-7710-8

(C) 1997 Springer Science+Business Media New York

Originally published by Plenum Press, New York in 1997

Softcover reprint of the hardcover 1st edition 1997

\section{1}

All rights reserved

No part of this book may be reproduced, stored in a retrieval system, or transmitted in any form or by any means, electronic, mechanical, photocopying, microfilming, recording, or otherwise, without written permission from the Publisher 


\section{Preface}

The term software engineering has been used extensively in different contexts. In a broader sense, software engineering encompasses every aspect of software systems; these include models, algorithms, programming, and management. Depending on one's interest, different perspectives of software engineering have been produced: project managers have emphasized managerial aspects of software products, programmers efficient programming, and computer scientists modeling and automating the programming process.

This book first introduces a number of software life cycle models and the basic concepts of object-oriented systems. Subsequently it discusses in more detail different phases of a software life cycle, with an emphasis on the objectoriented paradigm: "Formal Specification and Verification" (Chapter 3), "Design Methodologies and Specifications" (Chapter 4), "Programming and Coding" (Chapter 5), "Program Verification and Testing" (Chapter 9), and "Software Maintenance" (Chapter 10). Two of the six phases, namely, the requirement stage and the integration stage, are not covered extensively in separate chapters; rather in Chapter 1, several approaches to software integration are briefly discussed. It is my understanding that these two stages have been less understood in the past and more systematic approaches are yet to be developed. Several managerial issues related to software project management are covered in Chapter 12. Approaches to distributed software development are discussed in each stage whenever possible.

I cover the following subjects in more detail: "Programming Tools" (Chapter 6), "Declarative Programming" (Chapter 7), and "Automatic Program Synthesis and Reuse" (Chapter 8). The roles played by the preceding are shown graphically in the diagram below. With the aid of software libraries, programming tools allow a program to be composed with off-the-shelf modules, fully tested by experts, to increase the productivity of programmers and the quality of the software produced. Automatic program synthesis, although not a reality yet, can provide a shortcut from requirements specifications to maintenance. Declarative programming on the other hand is somewhat less ambitious but may relieve programmers of many implementation details. These chapters summarize the current status of such issues. Chapter 11 covers advanced software engineering environments that span several stages of the software development process; these 


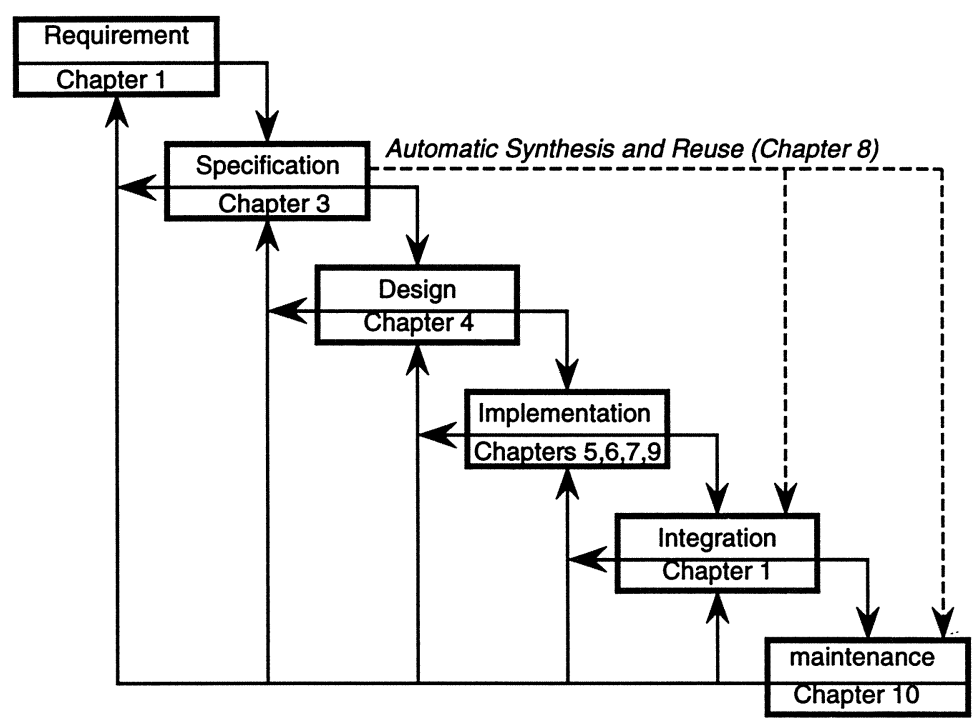

Scope of the book.

include knowledge-based programming environments, visual programming environments, distributed object-oriented programming environments, component software services, and concurrent engineering environments. Chapter 12 discusses issues of interest to software engineers; these include project management, fault-tolerant systems, discrete event simulation, and Internet programming.

I wish to thank Drs. C. V. Ramamoorthy, D. Cooke, R. Reynolds, L. J. Peterson, H. Freeman, T. Kidd, R. L. Kashyap, K. H. Kim, K. C. Tai, F. Calliss, R. DeMillo, P. Dewan, J. Riedl, R. Gordon, D. Yu, D. Lai, and W. T. Tsai for carefully reviewing this book and providing me with many insightful comments.

Although I tried my best to make this book an ideal one, I am sure it is not free of errors, typos, or omissions. I will appreciate it if the reader kindly lets me know of them. Suggestions for improvements will be deeply appreciated as well.

P. C.-Y. Sheu

Irvine, California 


\section{Contents}

Chapter 1. Software Life Cycle Models $\ldots \ldots \ldots \ldots \ldots \ldots \ldots \ldots, 1$

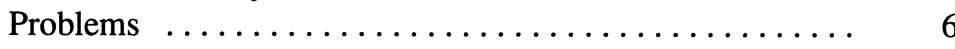

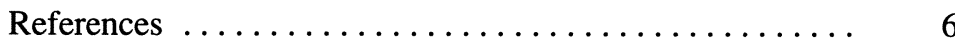

Chapter 2. Object-Oriented Concepts and Modeling ........... 9

2.1. Basic Concepts of the Object-Oriented Paradigm.... 10

2.1.1. Object, Class, Inheritance, and Message ..... 10

2.1.2. Object Identity ................... 10

2.1.3. Imperative and Active Objects.......... 11

2.1.4. Object-Oriented Libraries .............. 11

2.1.5. Object-Oriented Paradigm.............. 11

2.1.6. Examples ..................... 12

2.2. Object Modeling...................... 18

2.3. Advanced Object Models.................. 24

2.3.1. Real-Time Object Models.............. 24

2.3.2. Reflective Object-Oriented Models.......... 24

2.3.3. Agent-Based Object Models ............. 25

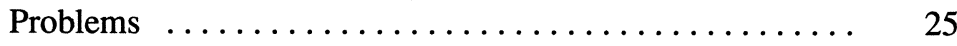

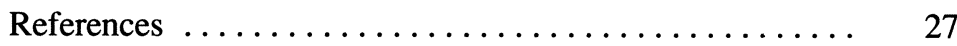

Chapter 3. Formal Specification and Verification ............ 29

3.1. First-Order Logic ...................... 29

3.1.1. First-Order Language $\ldots \ldots \ldots \ldots \ldots \ldots . \quad 30$

3.1.2. Semantics: Model and Interpretation ........ 30

3.1.3. First-Order Theory................. 31

3.1.4. Theorem Proving.................... 31

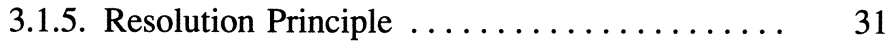

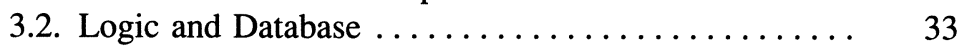

3.3. Formal Specification in Z $\ldots \ldots \ldots \ldots \ldots \ldots . \quad 35$

3.4. Object-Oriented Logic System............... 41

3.5. Algebraic Specifications................. 46

3.6. Petri Nets $\ldots \ldots \ldots \ldots \ldots \ldots \ldots \ldots \ldots . \ldots \ldots$ 
3.7. Verifying Specifications................ 52

3.8. Final Remarks....................... 60

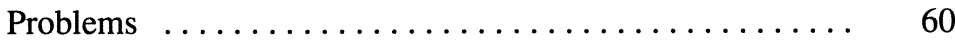

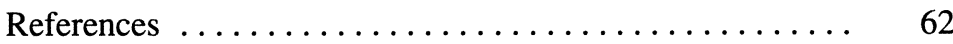

Chapter 4. Design Methodologies and Specifications .......... 65

4.1. Design Concepts..................... 65

4.2. Process Description Languages and Design

Methodologies....................... 67

4.2.1. Data Flow Diagrams and Design

Methodology ................... 67

4.2.2. SSL ....................... 72

4.2.3. HIPO $\ldots \ldots \ldots \ldots \ldots \ldots \ldots \ldots \ldots \ldots, 73$

4.2.4. INTERCOL $\ldots \ldots \ldots \ldots \ldots \ldots \ldots \ldots \ldots \ldots \ldots \ldots \ldots, 73$

4.2.5. Others..................... 76

4.3. Dynamic and Functional Modeling ........... 77

4.4. Object-Oriented Design Methodologies......... 80

4.5. Design Verification and CASE Tools ........... 86

Problems ............................. 89

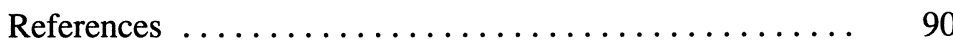

Chapter 5. Programming and Coding ................. 93

5.1. Object-Oriented Programming Languages........ 93

5.1.1. Objects ...................... 93

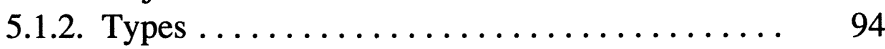

5.1.3. Inheritance ................... 94

5.1.4. Strongly Typed Object-Oriented Languages ... 95

5.1.5. Models of Concurrency................ 96

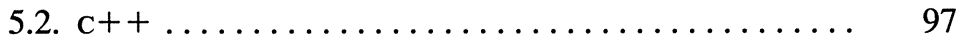

5.2.1. Program ..................... 98

5.2.2. Classes ....................... 98

5.2.3. Inheritance ................... 100

5.2.4. Operator Overloading ............... 103

5.3. SMALltalk ........................... 104

5.4. Distributed Programming Languages............ 109

5.4.1. Parallelism ....................... 110

5.4.2. Communication ................... 110

5.4.3. Synchronization .................. 111

5.4.4. Partial Failure/Fault Tolerance........... 111

5.4.5. Primitives for Parallelism ............. 113

5.4.6. Primitives for Communication and

Synchronization.................. 114 
5.4.7. Primitives for Partial Failure/Fault Tolerance .. 117

5.5. Communicating Sequential Processes and осСам .... 119

Problems ............................. 124

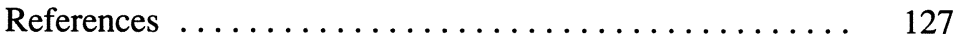

Chapter 6. Programming Tools ...................... 129

6.1. Object-Oriented Programming for Windows. . . . . . . 129

6.1.1. Simple Project . . . . . . . . . . . . . . . 130

6.1.2. Object Windows ................ 133

6.1.3. Sample Program ................ 138

6.2. IDE-An Interactive Programming Environment. . . . 140

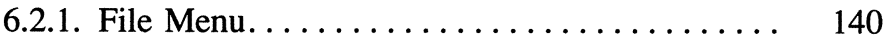

6.2.2. Edit Menu .................... 141

6.2.3. Compile Menu ..................... 141

6.2.4. Debug Menu .................. 142

6.2.5. Run Menu ................... 142

6.2.6. Search Menu ...................... 143

6.2.7. Option Menu . . . . . . . . . . . . . . . . . . 144

6.2.8. Project Menu . . . . . . . . . . . . . . . . 144

6.2.9. Window Menu .................... 145

6.3. X-Window Programming ................ 146

6.4. UNIX Network Programming . . . . . . . . . . . . . . 154

6.4.1. Simple Interprocess Communication ....... 155

6.4.2. Network Interprocess Programming . . . . . . . 157

Problems ......................... 163

References ....................... 180

Chapter 7. Declarative Programming .................. 181

7.1. Very High Level Programming ............. 181

7.2. Object-Oriented Declarative Programming ........ 183

7.3. Logic Programming-PROLOG.............. 190

7.4. COMPOSE-An Object-Oriented Programming

Environment . . . . . . . . . . . . . . . . . . . . . . . . . . . 194

7.4.1. Relational Query Languages . . . . . . . . . . . 194

7.4.2. COMPOSE. . . . . . . . . . . . . . . . . . . 198

Problems ........................... 205

References ...................... 206

Chapter 8. Automatic Program Synthesis and Reuse ........... 207

8.1. Deductive Program Synthesis . . . . . . . . . . . 207

8.1.1. Splitting Rules ... . . . . . . . . . . . . . . . 209

8.1.2. Transformation Rules ................. 209 
8.1.3. Resolution Rules .................. 210

8.1.4. Induction Hypothesis............... 211

8.2. Transformational Program Synthesis........... 216

8.3. Program Abstraction and Instantiation .......... 217

8.4. Automatic Software Reuse................. 220

Problems ........................... 227

References $\ldots \ldots \ldots \ldots \ldots \ldots \ldots \ldots \ldots \ldots \ldots, 228$

Chapter 9. Program Verification and Testing ............ 231

9.1. Formal Program Verification ............... 232

9.2. Black Box Testing ................... 235

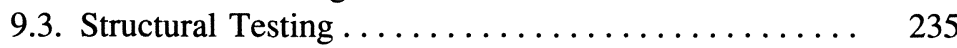

9.4. Error-Based Testing .................... 242

9.5. Automatic Test Case Generation............. 249

9.6. Testing and Debugging Distributed Programs ...... 251

9.7. Analysis Tools and Software Metrics ........... 255

9.7.1. Static Analysis Tools................ 255

9.7.2. Dynamic Analysis Tools.............. 256

9.7.3. Software Metrics ................. 257

Problems ......................... 258

References $\ldots \ldots \ldots \ldots \ldots \ldots \ldots \ldots \ldots \ldots \ldots .259$

Chapter 10. Software Maintenance ................. 261

10.1. Components of Software Maintenance ........ 261

10.1.1. Configuration Management............ 261

10.1.2. Version Management................ 262

10.2. Database and Software Maintenance......... 262

10.3. Programming Object Bases .............. 265

10.3.1. Queries...................... 269

10.3.2. Integrity Constraints ............. 270

10.3.3. Triggers $\ldots \ldots \ldots \ldots \ldots \ldots \ldots \ldots \ldots .270$

10.3.4. Views ...................... 270

10.4. Program Management................... 271

10.4.1. Incremental Testing............... 271

10.4.2. Integrity Control .................. 273

10.5. Reverse Engineering and Design Recovery ...... 274

10.5.1. Desire Version 1.0................ 277

10.5.2. MicroScope ....................... 277

Problems ............................ 279

References .......................... 279 
Chapter 11. Advanced Programming Environments. ......... 281

11.1. Knowledge-Based Programming Environments .... 281

11.1.1. KBEmacs .................... 281

11.1.2. CHI..................... 283

11.2. Visual Programming Environments.......... 284

11.3. Distributed Object-Oriented Programming Systems............................. 286

11.4. Component Software ................ 288

11.5. Programming Environments for Concurrent Engineering. ........................ 290

References .......................... 290

Chapter 12. Other Selected Topics ...................... 293

12.1. Project Management................... 293

12.1.1. Manager's Role .................. 293

12.1.2. Defining the Problem .............. 294

12.1.3. Proposals ....................... 294

12.1.4. Design ..................... 295

12.1.5. Programming ................... 295

12.1.6. System Testing ................. 296

12.1.7. Acceptance...................... 296

12.1.8. Operation ...................... 296

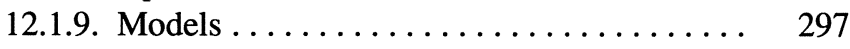

12.2. Fault-Tolerant System Design ............... 298

12.2.1. $N$-Version Programming ............ 299

12.2.2. Recovery Blocks .............. 300

12.2.3. Recovery Blocks versus $N$-Version Programming .................... 303

12.2.4. Designing with Fault Trees............ 304

12.3. Discrete Event Simulation ................. 305

12.4. Internet Programming. ............... 308

References $\ldots \ldots \ldots \ldots \ldots \ldots \ldots \ldots \ldots \ldots .6,316$

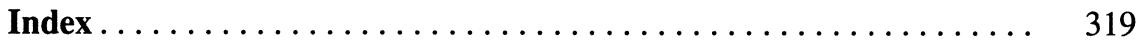

